\title{
Conductivity of pure graphene: Theoretical approach using the polarization tensor
}

\author{
G. L. Klimchitskaya ${ }^{1,2}$ and V. M. Mostepanenko ${ }^{1,2}$ \\ ${ }^{1}$ Central Astronomical Observatory at Pulkovo of the Russian \\ Academy of Sciences, Saint Petersburg, 196140, Russia \\ ${ }^{2}$ Institute of Physics, Nanotechnology and Telecommunications, \\ Peter the Great Saint Petersburg Polytechnic University, St.Petersburg, 195251, Russia
}

\begin{abstract}
We obtain analytic expressions for the conductivity of pristine (pure) graphene in the framework of the Dirac model using the polarization tensor in $(2+1)$-dimensions defined along the real frequency axis. It is found that at both zero and nonzero temperature $T$ the in-plane and outof-plane conductivities of graphene are equal to each other with a high precision and essentially do not depend on the wave vector. At $T=0$ the conductivity of graphene is real and equal to $\sigma_{0}=e^{2} /(4 \hbar)$ up to small nonlocal corrections in accordance with many authors. At some fixed $T \neq 0$ the real part of the conductivity varies between zero at low frequencies $\omega$ and $\sigma_{0}$ for optical $\omega$. If $\omega$ is fixed, the conductivity varies between $\sigma_{0}$ at low $T$ and zero at high $T$. The imaginary part of the conductivity of graphene is shown to depend on the ratio of $\omega$ to $T$. In accordance to the obtained asymptotic expressions, at fixed $T$ it varies from infinity at $\omega=0$ to a negative minimum value reached at some $\omega$, and then approaches to zero with further increase of $\omega$. At fixed $\omega$ the imaginary part of the conductivity varies from zero at $T=0$, reaches a negative minimum at some $T$ and then goes to infinity together with $T$. The numerical computations of both the real and imaginary parts of the conductivity are performed. The above results are obtained in the framework of quantum electrodynamics at nonzero temperature and can be generalized for graphene samples with nonzero mass gap parameter and chemical potential.
\end{abstract}

PACS numbers: 72.80.Vp, 73.63.-b, 65.80.Ck 


\section{INTRODUCTION}

It is generally recognized that graphene and other two-dimensional materials have opened up new fields in both fundamental and applied research. ${ }^{1}$ Unlike ordinary three-dimensional materials, quasiparticles in pure graphene are massless charged fermions. At energies below a few $\mathrm{eV}$ they possess the linear dispersion relation and obey the Dirac equation, where the speed of light $c$ is replaced with the Fermi velocity $v_{F} \approx c / 300$. This makes possible to use graphene for testing the Klein paradox, $\stackrel{2}{2}$ the effect of particle creation from vacuum by electric field, $\underline{3}^{-\underline{5}}$ the Casimir effect ${ }^{6,7}$ and other phenomena of relativistic quantum field theory.

Numerous applications of graphene in condensed matter physics and nanotechnology are based on its unusual electronic properties. $\stackrel{-8}{-}$ Among them the electrical conductivity of graphene has received the most study both theoretically and experimentally. $\underline{\underline{9}-11}$ The striking result from the study of conductivity of Dirac fermions ${ }^{12}$ is the existence of universal and frequency independent conductivity of graphene

$$
\sigma_{0}=\frac{\pi e^{2}}{2 h}=\frac{e^{2}}{4 \hbar}
$$

in the limiting case of vanishing temperature and disorder. It was noted also $\frac{12}{2}$ that if the frequency vanishes first and disorder second, the obtained minimal dc conductivity is different from $\sigma_{0}$. In this case for graphene one obtains $\sigma_{\mathrm{dc}}=4 e^{2} /(\pi h)=8 \sigma_{0} / \pi^{2}$. The existence of nonzero universal conductivity $\sigma_{0}$ is usually considered as surprising because in the limit of zero temperature there are no charge carriers, no scattering and no dissipation processes. Theoretically the conductivity of graphene was investigated in many papers using the current-current correlation functions, the Kubo formalism and Boltzmann's transport theory. $\underline{13-28}$ It was noted $\underline{11}, 21$ that the values of minimal conductivity obtained by different authors vary depending on the order of limiting transitions used in different theoretical approaches. At the same time, measurements of the conductivity of graphene ${ }^{29}-33$ result in somewhat larger values than the theoretical predictions. $\underline{11,22}$

During the last few years much attention was attracted also to investigation of the Casimir force between two graphene sheets, a graphene sheet and a plate made of ordinary material and between graphene-coated substrates. In so doing, the density-density correlation functions of graphene in the random phase approximation, the spatially nonlocal dielectric permittivities and other calculation methods have been used. $\underline{\underline{6}-3{ }^{-38}}$ It was shown, however, 
that the most complete results for the Casimir force in graphene systems are obtained using the polarization tensor of graphene in $(2+1)$-dimensional space-time. ${ }^{39}, \underline{40}$ Using this tensor defined at pure imaginary Matsubara frequencies, the Casimir effect in many graphene systems has been investigated $\underline{\underline{41}-\underline{46}}$ and an equivalence to some other calculation methods has been proved. $\underline{47}$

It should be taken into account that thermal quantum field theory provides the rigorous and straightforward formalism allowing calculation of the polarization tensor for different systems. Because of this, it is desirable to investigate as many physical phenomena as possible using this approach. Keeping in mind that calculation of the Casimir force in the framework of the Lifshitz theory $\underline{\underline{48}}$ is based on the reflection coefficients, it was interesting to study the reflectivity properties of graphene using the polarization tensor. For this purpose it was necessary to obtain an alternative representation for the polarization tensor of graphene valid not only at the pure imaginary Matsubara frequencies (as the one of Ref. [40]), but along the real frequency axis as well. This problem was solved in Ref. [49], and the reflectivity properties of pure graphene were studied over the wide range of frequencies. The obtained representation for the polarization tensor was also used to investigate the reflectivity properties of gapped graphene, graphene-coated substrates, $\frac{50}{\underline{w}}$ and large thermal effect in the Casimir force between two graphene sheets. $\underline{51,52}$

One further physical quantity of great interest directly expressed via the polarization tensor is the electrical conductivity. There are lots of papers in the literature where the conductivity of graphene is studied using different theoretical approaches (some of them are cited above). However, even for the case of pure graphene, where the effects of disorder are not taken into account, different values of the conductivity were obtained. Keeping in mind that the most of previous literature on the subject used various phenomenological models, it is reasonable to investigate the conductivity of pure graphene again starting from the first principles of thermal quantum field theory. In this paper we calculate both the longitudinal (in-plane) and transverse (out-of-plane) conductivities of pure (pristine) graphene using the formalism of the polarization tensor at arbitrary temperature. We obtain general analytic expressions and approximate asymptotic results for both the real and imaginary parts of the conductivity of graphene. The analytic results are accompanied by numerical computations over the wide regions of frequency and temperature. It is shown that at zero temperature the conductivity of graphene is real and takes the universal value $\sigma_{0}$ up to small nonlocal 
corrections. The real part of the conductivity of graphene taken at fixed temperature goes to zero and to $\sigma_{0}$ with decreasing and increasing frequency, respectively. We also show that the real part of the graphene conductivity at fixed frequency goes to $\sigma_{0}$ when the temperature vanishes and to zero with increasing temperature. At any nonzero temperature the conductivity of graphene has both the real and imaginary parts. We demonstrate that at some relationship between frequency and temperature the imaginary part of the conductivity of graphene drops to zero. At fixed temperature, the imaginary part of the conductivity goes to infinity with vanishing frequency and to zero with increasing frequency. At fixed frequency, the imaginary part of the graphene conductivity goes to zero with vanishing temperature and to infinity with increasing temperature. We also compare the obtained results with those found in the literature using some other theoretical approaches.

The paper is organized as follows. In Sec. II the general formalism connecting the conductivity of graphene with the polarization tensor is presented. In Sec. III the real part of the conductivity of graphene is investigated at both zero and nonzero temperature. Section IV is devoted to the imaginary part of the graphene conductivity. It presents both the asymptotic expressions and numerical results. In Sec. V the reader will find our conclusions and discussion.

Throughout the paper we preserve the fundamental constants in all formulas, but measure frequencies in the units of energy.

\section{GENERAL FORMALISM}

The polarization tensor of graphene at temperature $T$ in the one-loop approximation is defined as 40,49

$$
\begin{aligned}
\Pi_{\mu \nu}(\omega, \boldsymbol{k}, T) & =-8 \pi e^{2} \frac{k_{B} T}{\hbar c^{2}} \\
\times & \sum_{n=-\infty}^{\infty} \int \frac{d \boldsymbol{q}}{(2 \pi)^{2}} \operatorname{tr} S\left(q_{n}\right) \tilde{\gamma}_{\mu} S\left(q_{n}-k\right) \tilde{\gamma}_{\nu}
\end{aligned}
$$

where $k_{B}$ is the Boltzmann constant, $e$ is the electron charge, the trace is taken over the $\gamma$ matrices, and the spinor propagator is given by

$$
S(q)=\frac{1}{i \tilde{\gamma}_{\alpha} q^{\alpha}-m c / \hbar} .
$$


In Eq. (2) $k \equiv k^{\alpha}=(\omega / c, \boldsymbol{k})$ is the 3-dimensional wave vector of an external photon, where $\boldsymbol{k}=\left(k^{1}, k^{2}\right)$ are the components of the wave vector in the plane of graphene, and $q \equiv q^{\alpha}=\left(q^{0}, \boldsymbol{q}\right)$ is the 3 -dimensional wave vector of a loop electronic excitation. In the framework of the thermal quantum field theory in the Matsubara formulation the continuous zeroth component of the electronic wave vector should be replaced with the discrete halfinteger pure imaginary quantity

$$
q_{n}^{0}=2 \pi i \frac{k_{B} T}{c \hbar}\left(n+\frac{1}{2}\right), \quad q_{n} \equiv q_{n}^{\alpha}=\left(q_{n}^{0}, \boldsymbol{q}\right) .
$$

The $\tilde{\gamma}$ matrices in Eqs. (2) and (3) are connected with the standard $4 \times 4$ Dirac $\gamma$-matrices by the relations

$$
\tilde{\gamma}_{\alpha}=\eta_{\alpha}^{\beta} \gamma_{\beta}, \quad \eta_{\alpha}^{\beta} \equiv \operatorname{diag}\left(1, \tilde{v}_{F}, \tilde{v}_{F}\right),
$$

where $\tilde{v}_{F}=v_{F} / c$ and all the Greek indices here and above take the values $0,1,2$.

It is important that all components of the polarization tensor of graphene can be expressed $\underline{\underline{40}}$ via $\Pi_{00}$ and $\Pi_{\mathrm{tr}} \equiv \Pi_{\mu}^{\mu}$. Below it is more convenient to use the following combination:

$$
\Pi(\omega, \boldsymbol{k}, T) \equiv k^{2} \Pi_{\mathrm{tr}}(\omega, \boldsymbol{k}, T)+\left(\frac{\omega^{2}}{c^{2}}-k^{2}\right) \Pi_{00}(\omega, \boldsymbol{k}, T)
$$

instead of $\Pi_{\mathrm{tr}}$, where, starting from here, $k \equiv|\boldsymbol{k}|=\sqrt{k_{1}^{2}+k_{2}^{2}}$, i.e., is the magnitude of the projection of the photon wave vector on the plane of graphene. It was shown $\frac{40,49}{4}$ that the components of the polarization tensor depend only on $\omega, k=|\boldsymbol{k}|$ and $T$.

There are the familiar expressions connecting the in-plane $\left(\sigma_{\|}\right)$and out-of-plane $\left(\sigma_{\perp}\right)$ conductivities of the two-dimensional graphene sheet with its dynamical polarizabilities and dielectric permittivities $\underline{37}, \underline{53}$

$$
\alpha_{\|(\perp)}(\omega, k, T) \equiv \varepsilon_{\|(\perp)}(\omega, k, T)-1=\frac{2 \pi i \sigma_{\|(\perp)}(\omega, k, T) k}{\omega} .
$$

Using the Maxwell equations and the standard electrodynamic boundary conditions, one can express $9, \frac{37,54}{3}$ the amplitude reflection coefficients on the surface of graphene via $\alpha_{\|(\perp)}(\omega, k, T)$ or $\sigma_{\|(\perp)}(\omega, k, T)$ for the two independent polarizations of the electromagnetic field, transverse magnetic and transverse electric (i.e., for $p$ and $s$ polarizations). The Maxwell equations allow one to express the same reflection coefficients in terms of the polarization tensor $\underline{\underline{39}} \underline{\underline{41}}$ By comparing both exact expressions for the TM and TE reflection coefficients, the conductivity of graphene was expressed via the components of polarization 
tensor. 47 sing the representation for this tensor defined over the entire plane of complex frequencies, $\underline{49}$ rather than that valid at the Matsubara frequencies alone, $\underline{40}$ the conductivity of graphene takes the form

$$
\begin{aligned}
& \sigma_{\|}(\omega, k, T)=-i \frac{\omega}{4 \pi \hbar k^{2}} \Pi_{00}(\omega, k, T), \\
& \sigma_{\perp}(\omega, k, T)=i \frac{c^{2}}{4 \pi \hbar k^{2} \omega} \Pi(\omega, k, T) .
\end{aligned}
$$

It is convenient to represent the polarization tensor as a sum of the zero-temperature contribution and the thermal correction to it

$$
\begin{aligned}
& \Pi_{00}(\omega, k, T)=\Pi_{00}^{(0)}(\omega, k)+\Delta_{T} \Pi_{00}(\omega, k, T), \\
& \Pi(\omega, k, T)=\Pi^{(0)}(\omega, k)+\Delta_{T} \Pi(\omega, k, T) .
\end{aligned}
$$

The zero-temperature part can be written in the form 39,49

$$
\begin{aligned}
& \Pi_{00}^{(0)}(\omega, k)=i e^{2} \pi \frac{k^{2}}{\omega \eta(\omega, k)}, \\
& \Pi^{(0)}(\omega, k)=-i e^{2} \pi \frac{\omega}{c^{2}} k^{2} \eta(\omega, k),
\end{aligned}
$$

where

$$
\begin{aligned}
& \eta \equiv \eta(\omega, k)=\sqrt{1-\kappa^{2}(\omega, k)} \\
& \kappa \equiv \kappa(\omega, k)=\tilde{v}_{F} \frac{c k}{\omega} .
\end{aligned}
$$

Note that the zero-temperature contribution (10) is the same for both representations of the polarization tensor. $\stackrel{40,49}{\underline{4}}$ For real photons on a mass-shell $k \leq \omega / c$ and, thus, $\kappa \leq \tilde{v}_{F} \ll 1$.

The real part of the thermal correction to the polarization tensor ${ }^{49}$ can be most conveniently represented $\mathrm{as}^{50}$

$$
\begin{aligned}
& \operatorname{Re} \Delta_{T} \Pi_{00}(\omega, k, T)=\frac{8 e^{2} \omega}{\tilde{v}_{F}^{2} c^{2}} \sum_{j=1}^{3} Z_{00}^{(j)}(\omega, k, T), \\
& \operatorname{Re} \Delta_{T} \Pi(\omega, k, T)=\frac{8 e^{2} \omega^{3}}{\tilde{v}_{F}^{2} c^{4}} \sum_{j=1}^{3} Z^{(j)}(\omega, k, T) .
\end{aligned}
$$

Here, the three dimensionless quantities $Z_{00}^{(j)}$ are defined by

$$
\begin{aligned}
& Z_{00}^{(1)}(\omega, k, T) \equiv \int_{0}^{1-\kappa} \frac{d y}{e^{\beta y}+1}\left\{1-\frac{1}{2 \eta}\left[\sqrt{(y+1)^{2}-\kappa^{2}}+\sqrt{(y-1)^{2}-\kappa^{2}}\right]\right\} \\
& Z_{00}^{(2)}(\omega, k, T) \equiv \int_{1-\kappa}^{1+\kappa} \frac{d y}{e^{\beta y}+1}\left[1-\frac{1}{2 \eta} \sqrt{(y+1)^{2}-\kappa^{2}}\right], \\
& Z_{00}^{(3)}(\omega, k, T) \equiv \int_{1+\kappa}^{\infty} \frac{d y}{e^{\beta y}+1}\left\{1-\frac{1}{2 \eta}\left[\sqrt{(y+1)^{2}-\kappa^{2}}-\sqrt{(y-1)^{2}-\kappa^{2}}\right]\right\}
\end{aligned}
$$


where

$$
\beta \equiv \beta(\omega, T)=\frac{\omega}{2 \omega_{T}} \equiv \frac{\hbar \omega}{2 k_{B} T} .
$$

The quantity $\omega_{T}=k_{B} T / \hbar$ has the physical meaning of the thermal frequency. The three

dimensionless quantities $Z^{(j)}$ in Eq. (12) are obtained from the respective quantities $Z_{00}^{(j)}$ in Eq. (13) by the following substitution under the integrals:

$$
\sqrt{(y \pm 1)^{2}-\kappa^{2}} \rightarrow \frac{(y \pm 1)^{2}}{\sqrt{(y \pm 1)^{2}-\kappa^{2}}} .
$$

The imaginary part of the thermal correction to the polarization tensor ${ }^{49}$ can be rewritten in the form 50

$$
\begin{aligned}
& \operatorname{Im} \Delta_{T} \Pi_{00}(\omega, k, T)=\frac{4 e^{2} \omega}{\tilde{v}_{F}^{2} c^{2} \eta} Y_{00}(\omega, k, T), \\
& \operatorname{Im} \Delta_{T} \Pi(\omega, k, T)=-\frac{4 e^{2} \omega^{3} \eta}{\tilde{v}_{F}^{2} c^{4}} Y(\omega, k, T),
\end{aligned}
$$

where the dimensionless integrals are given by

$$
\begin{aligned}
& Y_{00}(\omega, k, T)=-\int_{1-\kappa}^{1+\kappa} \frac{d y}{e^{\beta y}+1} \sqrt{\kappa^{2}-(1-y)^{2}}, \\
& Y(\omega, k, T)=-\int_{1-\kappa}^{1+\kappa} \frac{d y}{e^{\beta y}+1} \frac{(1-y)^{2}}{\sqrt{\kappa^{2}-(1-y)^{2}}} .
\end{aligned}
$$

Here we present also an important property of the nonlocal dielectric permittivities 55

$$
\frac{\omega^{2}}{c^{2}} \lim _{k \rightarrow 0} \frac{\varepsilon_{\perp}(\omega, k, T)-\varepsilon_{\|}(\omega, k, T)}{k^{2}}=1-\frac{1}{\mu(\omega, T)},
$$

where $\mu(\omega, T)$ is the magnetic permeability of a medium. By putting in our case $\mu=1$ and using Eq. (77), one obtains

$$
\lim _{k \rightarrow 0} \frac{\alpha_{\perp}(\omega, k, T)-\alpha_{\|}(\omega, k, T)}{k^{2}}=0 .
$$

Below we use this equality, which means that for the normal incidence the response functions to $p$ and $s$ polarized light must be equal, to check a consistency of the obtained analytic expressions.

\section{REAL PART OF CONDUCTIVITY}

In this section the real part of the conductivity of pure graphene is investigated with the help of Eqs. (으)-(11), (16) and (17) expressing it via the polarization tensor. We start from the case of zero temperature and consider next the properties of the real part of conductivity at any temperature. 


\section{A. Conductivity via the polarization tensor at zero temperature}

As is seen from Eq. (10), the polarization tensor at $T=0$ is pure imaginary. Substituting Eq. (10) in Eq. (8), one arrives to the longitudinal and transverse real conductivities of graphene at zero temperature

$$
\begin{aligned}
& \sigma_{\|}(\omega, k, 0)=\frac{e^{2}}{4 \hbar \eta(\omega, k)}=\frac{\sigma_{0}}{\sqrt{1-\kappa^{2}(\omega, k)}}, \\
& \sigma_{\perp}(\omega, k, 0)=\frac{e^{2}}{4 \hbar} \eta(\omega, k)=\sigma_{0} \sqrt{1-\kappa^{2}(\omega, k)}
\end{aligned}
$$

where $\sigma_{0}$ is expressed in Eq. (1). The same expressions for $\sigma_{\|(\perp)}$ are presented in Ref. [56] which contains a summary of the obtained results at $T=0$ and nonzero chemical potential. Taking into account Eq. (11) and expanding in powers of a small parameter $\kappa$, one arrives at

$$
\begin{aligned}
& \sigma_{\|}(\omega, k, 0) \approx \sigma_{0}\left[1+\frac{1}{2} \tilde{v}_{F}^{2}\left(\frac{c k}{\omega}\right)^{2}+O\left(\tilde{v}_{F}^{4}\right)\right], \\
& \sigma_{\perp}(\omega, k, 0) \approx \sigma_{0}\left[1-\frac{1}{2} \tilde{v}_{F}^{2}\left(\frac{c k}{\omega}\right)^{2}+O\left(\tilde{v}_{F}^{4}\right)\right] .
\end{aligned}
$$

As is seen from Eq. (21), at $T=0$ both conductivities of graphene are equal to a high precision to the universal constant conductivity $\sigma_{0}$. The same value for the minimal conductivity

of graphene was obtained by different authors using the Kubo formula. 12,18,19,21,28,56,57 The nonlocal corrections to this result depending on $\omega$ and $k$ are of the order of $10^{-5}$ and can be neglected.

It is easy to check the fulfilment of Eq. (19) at $T=0$. For this purpose we substitute Eq. (10) in Eq. (7) and obtain

$$
\alpha_{\|}(\omega, k, 0)=i \frac{e^{2} \pi}{2 \hbar} \frac{k}{\omega \sqrt{1-\kappa^{2}}}, \quad \alpha_{\perp}(\omega, k, 0)=i \frac{e^{2} \pi}{2 \hbar} \frac{k}{\omega} \sqrt{1-\kappa^{2}} .
$$

From these equations we get

$$
\alpha_{\perp}(\omega, k, 0)-\alpha_{\|}(\omega, k, 0)=-i \frac{e^{2} \pi c^{2}}{2 \hbar} \frac{k^{3}}{\omega^{3}} \frac{\tilde{v}_{F}^{2}}{\sqrt{1-\kappa^{2}}} .
$$

Taking into account that $\kappa \rightarrow 0$ when $k \rightarrow 0$, we find that Eq. (19) is satisfied. 


\section{B. Real part of conductivity via the polarization tensor at nonzero temperature}

As shown in Sec. II, at nonzero temperature the polarization tensor has both the real and imaginary parts. The real part of the conductivity under consideration in this section is determined by the imaginary of the polarization tensor presented in Eq. (16). We start with analytic expressions for the real part of the conductivity of graphene and then continue with the results of numerical computations.

\section{Analytic expressions for the real part of conductivity}

According to Eqs. (8) -(10) the real parts of the longitudinal and transverse conductivities of graphene at nonzero temperature are given by

$$
\begin{aligned}
& \operatorname{Re} \sigma_{\|}(\omega, k, T)=-i \frac{\omega}{4 \pi \hbar k^{2}}\left[\Pi_{00}^{(0)}(\omega, k)+i \operatorname{Im} \Delta_{T} \Pi_{00}(\omega, k, T)\right], \\
& \operatorname{Re} \sigma_{\perp}(\omega, k, T)=i \frac{c^{2}}{4 \pi \hbar k^{2} \omega}\left[\Pi^{(0)}(\omega, k)+i \operatorname{Im} \Delta_{T} \Pi(\omega, k, T)\right] .
\end{aligned}
$$

Substituting Eqs. (10) and (16) in Eq. (24), one obtains

$$
\begin{aligned}
& \operatorname{Re} \sigma_{\|}(\omega, k, T)=\frac{\sigma_{0}}{\eta}\left[1+\frac{4}{\pi \kappa^{2}} Y_{00}\right], \\
& \operatorname{Re} \sigma_{\perp}(\omega, k, T)=\sigma_{0} \eta\left[1+\frac{4}{\pi \kappa^{2}} Y\right],
\end{aligned}
$$

where the integrals $Y_{00}$ and $Y$ are defined in Eq. (17).

It is convenient to introduce the new variable $t=(1-y) / \kappa$ in both integrals in Eq. (17). In terms of the new variable these integrals take the form

$$
\begin{aligned}
& Y_{00}=-\kappa^{2} \int_{-1}^{1} \frac{d t}{e^{\beta} e^{-\beta \kappa t}+1} \sqrt{1-t^{2}}, \\
& Y=-\kappa^{2} \int_{-1}^{1} \frac{d t}{e^{\beta} e^{-\beta \kappa t}+1} \frac{t^{2}}{\sqrt{1-t^{2}}} .
\end{aligned}
$$

Taking into account Eqs. (11) and (14), for the parameter $\beta \kappa$ we have

$$
\beta \kappa=\frac{c \tilde{v}_{F} \hbar k}{2 k_{B} T} \leq \tilde{v}_{F} \frac{\hbar \omega}{2 k_{B} T} .
$$

As a result, the integrals (26) can differ from zero only in the region of the plane $(\omega, T)$ where $\beta \kappa \ll 1$. This is apparent from the fact that, for example, for $\beta \kappa>0.1$ it occurs

$$
0.1<\beta \kappa<\tilde{v}_{F} \beta
$$


and, thus, $\beta>30$. In this case both functions under the integrals in Eq. (26) are of the order of $\exp (-30)$.

We arrive at the conclusion that in the region of $(\omega, T)$ plane, where the thermal correction contributes to the result, the function under the integrals in Eq. (26) containing exponents can be expanded in powers of the small parameter $\beta \kappa$ :

$$
\begin{array}{r}
\frac{1}{e^{\beta} e^{-\beta \kappa t}+1}=\frac{1}{e^{\beta}+1}+\beta \kappa \frac{e^{-\beta}}{\left(1+e^{-\beta}\right)^{2}} t \\
+(\beta \kappa)^{2} \frac{e^{-\beta}\left(1-e^{-\beta}\right)}{2\left(1+e^{-\beta}\right)^{3}} t^{2}+O\left[(\beta \kappa)^{3}\right] .
\end{array}
$$

Substituting this in the first line of Eq. (26) and performing the integration, we arrive at

$$
Y_{00}=-\kappa^{2}\left[\frac{\pi}{2\left(e^{\beta}+1\right)}+\kappa^{2} \frac{\pi \beta^{2} e^{-\beta}\left(1-e^{-\beta}\right)}{16\left(1+e^{-\beta}\right)^{3}}+O\left(\kappa^{4}\right)\right] .
$$

Note that the terms in Eq. (29) containing the odd powers of the small parameter $\beta \kappa$ (and, thus, the odd powers of $t$ ) do not contribute to the result (30). In a similar way, substituting Eq. (29) in the second line of Eq. (26) and integrating, we find

$$
Y=-\kappa^{2}\left[\frac{\pi}{2\left(e^{\beta}+1\right)}+\kappa^{2} \frac{3 \pi \beta^{2} e^{-\beta}\left(1-e^{-\beta}\right)}{16\left(1+e^{-\beta}\right)^{3}}+O\left(\kappa^{4}\right)\right] .
$$

Now we substitute Eqs. (30) and (31) in Eq. (25), expand $\eta$ in powers of a small parameter $\kappa$ defined in Eq. (11) and combine all terms with similar powers of $\kappa$. The result is

$$
\operatorname{Re} \sigma_{\|(\perp)}(\omega, k, T)=\sigma_{0}\left[1-\frac{2}{e^{\beta}+1}-\kappa^{2} C_{\|(\perp)}(\beta)+O\left(\kappa^{4}\right)\right],
$$

where the functions $C_{\|}$and $C_{\perp}$ are given by

$$
\begin{aligned}
& C_{\|}(\beta)=\frac{\beta^{2} e^{-\beta}\left(1-e^{-\beta}\right)+2\left(1+e^{-\beta}\right)^{2}\left(1+3 e^{-\beta}\right)}{4\left(1+e^{-\beta}\right)^{3}}, \\
& C_{\perp}(\beta)=\frac{3 \beta^{2} e^{-\beta}\left(1-e^{-\beta}\right)-2\left(1+e^{-\beta}\right)^{2}\left(1+3 e^{-\beta}\right)}{4\left(1+e^{-\beta}\right)^{3}} .
\end{aligned}
$$

According to Eq. (11), the dependence of the real parts of conductivities (32) on $k$ is contained only in the quantities $\kappa \leq \tilde{v}_{F}$, whereas according to Eq. (14) the dependence on $\omega$ and $T$ is determined by the parameter $\beta$. Taking into account that the relative contribution of the second order terms in Eq. (32) does not exceed $10^{-4}$, one arrives at ${ }^{54}$

$$
\operatorname{Re} \sigma_{\|(\perp)}(\omega, T) \approx \sigma_{0}\left[1-\frac{2}{e^{\hbar \omega /\left(2 k_{B} T\right)}+1}\right]=\sigma_{0} \tanh \frac{\hbar \omega}{4 k_{B} T} .
$$


The real part of $\sigma_{\|(\perp)}$ is approximately equal to $\sigma_{0}$ in the limiting case of high frequencies $\omega \gg 2 \omega_{T}$ and goes to zero in the limiting case of low frequencies $\omega \ll 2 \omega_{T}$.

Note that Eq. (34) possesses a discontinuity as a function of two variables $\omega$ and $T$ at the point $(0,0)$ :

$$
\begin{aligned}
& \lim _{\omega \rightarrow 0} \operatorname{Re} \sigma_{\|(\perp)}(\omega, T \neq 0)=0, \\
& \lim _{T \rightarrow 0} \operatorname{Re} \sigma_{\|(\perp)}(\omega \neq 0, T)=\sigma_{0} .
\end{aligned}
$$

We also note that the real part of the conductivity of graphene calculated using the polarization tensor does not contain a singular term which arises in theoretical approaches using the Drude model ${ }^{56}$ when the relaxation parameter goes to zero. This is because the Dirac model for pure graphene does not take into account the collisions of quasiparticles. In terms of the Drude model this means that the relaxation parameter is put equal to zero from the outset.

Finally, we check that the obtained results satisfy the condition (19). From Eq. (17) we have

$$
\operatorname{Re} \alpha_{\|(\perp)}=-2 \pi \frac{k}{\omega} \operatorname{Im} \sigma_{\|(\perp)}, \quad \operatorname{Im} \alpha_{\|(\perp)}=2 \pi \frac{k}{\omega} \operatorname{Re} \sigma_{\|(\perp)} .
$$

At this point we can use only the second equality in Eq. (36) leaving the first one for Sec. IV. Substituting Eq. (32) in Eq. (36), one finds

$$
\operatorname{Im} \alpha_{\|(\perp)}=2 \pi \frac{k}{\omega} \sigma_{0}\left[1-\frac{2}{e^{\beta}+1}-\kappa^{2} C_{\|(\perp)}+O\left(\kappa^{4}\right)\right] .
$$

As a result, using Eq. (11), we obtain

$$
\operatorname{Im} \alpha_{\perp}-\operatorname{Im} \alpha_{\|}=2 \pi \sigma_{0} \tilde{v}_{F}^{2} c^{2} \frac{k^{3}}{\omega^{3}}\left[C_{\|}-C_{\perp}\right]
$$

i.e., the condition (19) is satisfied.

\section{Numerical results}

We are coming now to numerical computations of the real part of the conductivity of graphene obtained using the polarization tensor over a wide range frequencies and temperatures. For this purpose one can use either the exact Eqs. (25) and (26) or the approximate Eqs. (32) and (33). In both cases the computational results are essentially independent on $k$ and, thus, the local limit $k=0$ can be taken from the very beginning. 
In Fig. 1 we plot the real part of the conductivity of graphene, normalized to the universal conductivity $\sigma_{0}$ defined in Eq. (11), as a function of frequency (we remind that $1 \mathrm{eV} \approx$ $\left.1.52 \times 10^{15} \mathrm{rad} / \mathrm{s}\right)$. The lines from bottom to top are computed for the values of temperature equal to $300 \mathrm{~K}, 100 \mathrm{~K}$ and $10 \mathrm{~K}$, respectively. As is seen in this figure, with increasing $\omega$ all lines go to a common universal limiting value $\sigma_{0}$ in accordance with Eq. (34). This value of the real part of the conductivity is already achieved at the frequency of about $0.01 \mathrm{eV}$ when the temperature is minimal $(T=10 \mathrm{~K})$. With increasing temperature to $100 \mathrm{~K}$ and $300 \mathrm{~K}$ the conductivity $\sigma_{0}$ is achieved for frequencies satisfying the inequalities $\omega>0.1 \mathrm{eV}$ and $\omega>0.25 \mathrm{eV}$, respectively. We note that at $T=10 \mathrm{~K}\left(2 k_{B} T \approx 0.0016 \mathrm{eV}\right)$ the maximum value of the parameter (14) is

$$
\beta_{\max }=\frac{\hbar \omega_{\max }}{2 k_{B} T}=\frac{0.25}{0.0016} \approx 150 .
$$

Taking into account that $\kappa_{\max }=\tilde{v}_{F}$, one obtains $\beta_{\max } \kappa_{\max } \approx 0.5$, i.e., in the discussed above region $\beta \kappa>0.1$, where the thermal corrections are negligibly small in accordance with the top line in Fig. 1. With increasing $T$ the parameter $\beta$ decreases and the role of thermal effects becomes important over the wider ranges of frequencies (see the middle and bottom lines in Fig. (1).

With decreasing $\omega$ the real part of the conductivity of graphene approaches zero in accordance with Eq. (34). This approach becomes slower with increasing temperature. Our computational results for the real part of the conductivity of graphene in Fig. 1 are in agreement with those obtained using the tight-binding model $\underline{58}$

Now we compute the real part of the conductivity of graphene at fixed frequency as a function of temperature. The computational results normalized to the universal conductivity $\sigma_{0}$ are presented in Fig. 2(a) in the temperature region from $0.5 \mathrm{~K}$ to room temperature. The lines 1, 2, 3, and 4 correspond to frequencies $\omega$ equal to $0.1,0.01,0.001$, and $0.0001 \mathrm{eV}$, respectively. As is seen in Fig. 2(a), with increasing temperature the real part of the graphene conductivity at all frequencies decreases and goes to the common value equal to zero. This decrease is very slow at the largest frequency and becomes pronounced with decreasing frequency. For better visualization of the region of low temperatures, in Fig. 2(b) we plot the same lines on an enlarged scale in the vicinity of the absolute zero (here the lines 1 and 2 overlap). As is seen in Fig. 2(b), the real part of the conductivity of graphene goes to $\sigma_{0}$ with vanishing temperature. Thus, Figs. 1 and $2(a, b)$ illustrate the discontinuity of the real 
part of graphene conductivity (35) as a function of frequency and temperature.

\section{IMAGINARY PART OF CONDUCTIVITY}

Here, we consider the imaginary part of the conductivity of graphene which exists only at nonzero temperature. In our formalism it is expressed via the real part of the polarization tensor presented in Eqs. (12)-(15). We start with the asymptotic expressions for the imaginary part of the graphene conductivity and continue with the results of numerical computations.

\section{A. Asymptotic expressions at low and high frequencies}

Taking into account Eqs. (8) -(10), the imaginary parts of the conductivities of graphene are given by

$$
\begin{aligned}
& \operatorname{Im} \sigma_{\|}(\omega, k, T)=-\frac{\omega}{4 \pi \hbar k^{2}} \operatorname{Re} \Delta_{T} \Pi_{00}(\omega, k, T), \\
& \operatorname{Im} \sigma_{\perp}(\omega, k, T)=\frac{c^{2}}{4 \pi \hbar k^{2} \omega} \operatorname{Re} \Delta_{T} \Pi(\omega, k, T) .
\end{aligned}
$$

Substituting Eq. (12) in Eq. (40), one obtains

$$
\begin{aligned}
& \operatorname{Im} \sigma_{\|}(\omega, k, T)=-\sigma_{0} \frac{8}{\pi \kappa^{2}} \sum_{j=1}^{3} Z_{00}^{(j)}(\omega, k, T), \\
& \operatorname{Im} \sigma_{\perp}(\omega, k, T)=\sigma_{0} \frac{8}{\pi \kappa^{2}} \sum_{j=1}^{3} Z^{(j)}(\omega, k, T),
\end{aligned}
$$

where the integrals $Z_{00}^{(j)}$ and $Z^{(j)}$ are defined in Eqs. (13)-(15).

Now we consider the case of low frequencies, as compared to the thermal frequency, i.e., $\omega \ll 2 \omega_{T}(\beta \ll 1)$. In this case the asymptotic expressions for the real part of the polarization tensor have been obtained in the literature

$$
\begin{aligned}
& \operatorname{Re} \Delta_{T} \Pi_{00}(\omega, k, T)=-8 \frac{e^{2}}{\hbar} \ln 2 \frac{k_{B} T k^{2}}{\omega^{2}}+O\left(\tilde{v}_{F}^{2} k^{4}\right) \\
& \operatorname{Re} \Delta_{T} \Pi(\omega, k, T)=8 \frac{e^{2}}{\hbar} \ln 2 \frac{k_{B} T k^{2}}{c^{2}}+O\left(\tilde{v}_{F}^{2} k^{4}\right)
\end{aligned}
$$

(see Eqs. (88) and (95) in Ref. [49] and Eq. (19) in Ref. [50]). Note that in this case of low frequencies only the integrals $Z_{00}^{(3)}$ and $Z^{(3)}$ in Eq. (41) give the dominant contribution, 
whereas the other integrals are negligibly small. Using Eq. (42) we find the common result for the imaginary parts of both conductivities

$$
\operatorname{Im} \sigma_{\|(\perp)}(\omega, k, T)=\sigma_{0} \frac{8 \ln 2}{\pi} \frac{k_{B} T}{\hbar \omega}+O\left(\tilde{v}_{F}^{2} k^{2}\right) .
$$

Under the condition $\hbar \omega \lesssim 0.2 k_{B} T$ the use of asymptotic expression (43) results in less than $1 \%$ error in the obtained results.

We continue with the case of high frequencies satisfying the condition $\omega \gg 2 \omega_{T}(\beta \gg 1)$. In this case the asymptotic expressions for the real part of the polarization tensor is contained in Eqs. (67) and (76) in Ref. [49]:

$$
\begin{aligned}
& \operatorname{Re} \Delta_{T} \Pi_{00}(\omega, k, T)=48 \zeta(3) e^{2} \frac{k^{2}\left(k_{B} T\right)^{3}}{\hbar^{3} \omega^{4}}+O\left(\tilde{v}_{F}^{2} k^{4}\right), \\
& \operatorname{Re} \Delta_{T} \Pi(\omega, k, T)=-48 \zeta(3) e^{2} \frac{k^{2}\left(k_{B} T\right)^{3}}{c^{2} \hbar^{3} \omega^{2}}+O\left(\tilde{v}_{F}^{2} k^{4}\right),
\end{aligned}
$$

where $\zeta(z)$ is the Riemann zeta function. The results (44) are determined by the integrals $Z_{00}^{(1)}$ and $Z^{(1)}$ in Eq. (41). Then for the imaginary parts of the conductivities one obtains

$$
\operatorname{Im} \sigma_{\|(\perp)}(\omega, k, T)=-\sigma_{0} \frac{48 \zeta(3)}{\pi}\left(\frac{k_{B} T}{\hbar \omega}\right)^{3}+O\left(\tilde{v}_{F}^{2} k^{2}\right) .
$$

These expressions lead to a less than $1 \%$ error if $\hbar \omega>70 k_{B} T$.

It is possible now to check the fulfilment of the condition (19) for the real parts of the polarizabilities of graphene. From the first equality in Eq. (36), using Eq. (43) obtained under the condition $\omega \ll 2 \omega_{T}$, one finds

$$
\operatorname{Re} \alpha_{\|(\perp)}=-4 e^{2} \ln 2 \frac{k_{B} T}{(\hbar \omega)^{2}} k+O\left(\tilde{v}_{F}^{2} k^{3}\right)
$$

From this equation we immediately arrive at $\operatorname{Re}\left(\alpha_{\perp}-\alpha_{\|}\right)=O\left(\tilde{v}_{F}^{2} k^{3}\right)$ and Eq. (19) is satisfied.

In a similar way, under the condition $\omega \gg 2 \omega_{T}$ from Eqs. (36) and Eq. (45) one finds

$$
\operatorname{Re} \alpha_{\|(\perp)}=24 \zeta(3) e^{2} \frac{\left(k_{B} T\right)^{3}}{(\hbar \omega)^{4}} k+O\left(\tilde{v}_{F}^{2} k^{3}\right) .
$$

From Eq. (47) we again conclude that Eq. (19) is satisfied.

\section{B. Numerical results}

Numerical computations of the imaginary part of the conductivity of graphene were performed by Eq. (41), where all notations are introduced in Eqs. (13)-(15). Numerical 
integrations were performed at different values of $k$ entering Eqs. (13)-(15) and (41) only through the quantity $\kappa$. Specifically, within the intervals of $\kappa / \tilde{v}_{F}$ from 1 to 0.001 and of $\beta$ from 0.0001 to 30, it was found that at each value of $\beta$ it occurs $\sigma_{\|} \approx \sigma_{\perp}$, and the obtained value of $\sigma_{\|(\perp)}$ does not depend on $\kappa$ within the relative error smaller than $0.05 \%$. This maximum error is achieved only in the narrow vicinity of the value $\beta=\beta_{0}$ where the imaginary part of the conductivity of graphene takes the zero value. Thus, for all practical purposes one can consider the longitudinal and transverse conductivities of graphene equal and compute them at any nonzero value of $k$ as a function of $\beta$ which is effectively equal to the ratio of frequency to twice temperature.

In Fig. 3 the computational results for the magnitude of the imaginary part of graphene conductivity normalized to $\sigma_{0}$ are shown by the solid line in the double logarithmic scale as a function of $\beta=\omega /\left(2 \omega_{T}\right)$. In the same figure the left and right dashed lines present the asymptotic results at low and high frequencies given by Eqs. (43) and (45), respectively. As is seen in Fig. 3, at both low and high frequencies the analytic asymptotic expressions in Eqs. (43) and (45) are in a very good agreement with the results of numerical computations using the exact formula (41). It is also seen that at some ratio of frequency to temperature $\beta_{0}$ the imaginary part of the conductivity of graphene takes the zero value.

In Fig. 4 we present the imaginary part of the graphene conductivity normalized to $\sigma_{0}$ as a function of $\beta$ in a narrow vicinity of the point $\beta_{0}$, where the imaginary part vanishes. The computations were performed for $\kappa=0.01 \tilde{v}_{F}$ and result in $\beta_{0}=2.077563$. As was noted above, this result does not depend on the value $\kappa(k)$ to a high precision. As can be seen in Fig. 4, for $\beta<\beta_{0}$ the imaginary part of the conductivity of graphene is positive and decreases from infinity to zero with increasing $\beta$. For $\beta>\beta_{0}$ the imaginary part of the conductivity is negative and approaches zero with the increase of $\beta$. The behavior of $\operatorname{Im} \sigma_{\|(\perp)}$ in the limiting cases $\beta \rightarrow 0$ and $\beta \rightarrow \infty$ is in perfect agreement with the asymptotic expressions (43) and (45).

Next, we investigate the imaginary part of the conductivity of graphene at different fixed temperatures. In Fig. 5 the three lines from right to left present the computational results for $\operatorname{Im} \sigma_{\|(\perp)} / \sigma_{0}$ as the functions of frequency at the temperatures $T=300 \mathrm{~K}, 100 \mathrm{~K}$, and $10 \mathrm{~K}$, respectively. As is seen in Fig. 5, with decreasing temperature from room temperature to $10 \mathrm{~K}$, the value of frequency $\omega_{0}$, where the imaginary part of the conductivity vanishes, goes to zero, and the region of frequencies, where it takes positive values, becomes more narrow. 
Thus, at $T=300 \mathrm{~K}, 100 \mathrm{~K}$, and $10 \mathrm{~K}$, we have $\omega_{0}=0.103878,0.034626$, and $0.0034626 \mathrm{eV}$, respectively.

Finally, we compute the imaginary part of the graphene conductivity as a function of temperature. In Fig. [6(a) we plot $\operatorname{Im} \sigma_{\|(\perp)} / \sigma_{0}$ by the three lines numbered 1, 2, and 3 as the function of $T$ at the frequencies $\omega=0.1,0.01$, and $0.001 \mathrm{eV}$, respectively. As is seen in Fig. 6(a), at zero temperature the imaginary part of the conductivity is equal to zero, takes negative values with increasing $T$, changes sign at some $T=T_{0}$ and then goes to infinity with further increase of $T$. This is in perfect agreement with our asymptotic expressions if to take into account that the case of low $T$ corresponds to the asymptotic expression of high frequencies (45) because at low $T$ in holds $\hbar \omega /\left(2 k_{B} T\right) \gg 1$. In a similar way, at high $T$ the asymptotic expression of low frequencies (43) is applicable. With decreasing $T$ the value of $T_{0}$ decreases linearly. Thus, it is equal to $T_{0}=288,8 \mathrm{~K}, 28.88 \mathrm{~K}$, and $2.888 \mathrm{~K}$ at $\omega=0.1$, 0.01 , and $0.001 \mathrm{eV}$, respectively.

To illustrate the behavior of the imaginary part of the conductivity at low $T$, in Fig. 6(b) we again plot the lines 1-3 already plotted in Fig. 6(a), but now over the narrow temperature interval from 0 to $5 \mathrm{~K}$ and supplement them by the line 4 plotted at $\omega=10^{-4} \mathrm{eV}$. As is seen in Fig. 6(b), the lines 1 and 2 are now almost coinciding with the horizontal axis, whereas the line 4 demonstrates typical behavior of the imaginary part of the graphene conductivity as a function of temperature. For the line 4 we have $T_{0}=0.2888 \mathrm{~K}$.

\section{CONCLUSIONS AND DISCUSSION}

In the foregoing, we have investigated the conductivity of pristine (pure) graphene in the framework of the Dirac model using the formalism of the polarization tensor in $(2+1)$ dimensional space-time. Previously the conductivity of graphene was investigated using different formalisms and there was no complete agreement in the literature with respect to its minimal value. According to our results, both the longitudinal and transverse conductivities of pure graphene at zero temperature are equal to a high precision to $\sigma_{0}$ defined in Eq. (1) in agreement with a number of authors. $12,18,19,21,28,56,57$ We emphasize that the formalism of the polarization tensor is formulated starting from the first principles of thermal quantum field theory. It does not use any phenomenological model and the concept of disorder with subsequent limiting transition of the disorder parameter to zero. This in some sense simplifies 
calculation of the graphene conductivity and makes it more transparent.

We have found that the real part of the conductivity of graphene at fixed nonzero temperature goes to zero when the frequency vanishes and achieves $\sigma_{0}$ with increasing frequency. However, if the frequency is kept constant, the real part of the conductivity of graphene goes to $\sigma_{0}$ and zero with decreasing and increasing temperature, respectively. It is shown that in the local approximation the real parts of the longitudinal and transverse conductivities at $T \neq 0$ are equal, and that the corrections due to a nonlocality are negligibly small up to a very high precision. We have also obtained convenient exact analytic expressions for the real part of the graphene conductivity. Using these expressions, numerical computations of the real part of the conductivity of graphene have been performed.

In this paper, the exact analytic expressions for the imaginary part of the conductivity of graphene, which is not equal to zero at nonzero temperature, are obtained. The asymptotic expressions for the imaginary part of the graphene conductivity are found at both low and high frequencies, as compared to the thermal frequency. It is shown that the imaginary parts of longitudinal and transverse conductivities are equal and do not depend on the wave vector to a high precision. At some fixed temperature, the imaginary part of the conductivity goes to infinity when the frequency vanishes, takes the zero value at some frequency, changes its sign at higher frequencies and goes to zero with further increase of frequency. At fixed frequency the imaginary part of the conductivity takes the zero value at zero frequency, reaches some negative minimum value with increasing temperature and then goes to infinity when the temperature further increases.

The imaginary part of the conductivity of graphene is shown to depend on the ratio of $\omega$ to $2 T$. According to the obtained asymptotic expressions, at fixed $T$ it varies from infinity at $\omega=0$ to a negative minimum value at some fixed $\omega$, and increases to zero with further increase of $\omega$. At fixed $\omega$ the imaginary part of the conductivity varies from zero at $T=0$, reaches a negative minimum value at some $T$ and goes to infinity together with $T$. Numerical computations of the imaginary part of the conductivity are performed over the wide ranges of frequency and temperature.

In future it would be useful to extend the present analysis based on first principles of thermal quantum field theory to graphene with nonzero mass-gap parameter and chemical 
potential.

1 M. I. Katsnelson, Graphene: Carbon in Two Dimensions (Cambridge University Press, Cambridge, 2012).

2 M. I. Katsnelson, K. S. Novoselov, and A. K. Geim, Nature Phys. 2, 620 (2006).

3 D. Allor, T. D. Cohen, and D. A. McGady, Phys. Rev. D 78, 096009 (2008).

4 C. G. Beneventano, P. Giacconi, E. M. Santangelo, and R. Soldati, J. Phys A: Math. Theor. 42, 275401 (2009).

5 G. L. Klimchitskaya and V. M. Mostepanenko, Phys. Rev. D 87, 215011 (2013).

6 G. Gómez-Santos, Phys. Rev. B 80, 245424 (2009).

7 A. A. Banishev, H. Wen, J. Xu, R. K. Kawakami, G. L. Klimchitskaya, V. M. Mostepanenko, and U. Mohideen, Phys. Rev. B 87, 205433 (2013).

8 A. H. Castro Neto, F. Guinea, N. M. R. Peres, K. S. Novoselov, and A. K. Geim, Rev. Mod. Phys. 81, 109 (2009).

9 V. P. Gusynin, S. G. Sharapov, and J. P. Carbotte, Int. J. Mod. Phys. B 21, 4611 (2007).

10 N. M. R. Peres, Rev. Mod. Phys. 82, 2673 (2010).

11 S. Das Sarma, S. Adam, E. H. Hwang, and E. Rossi, Rev. Mod. Phys. 83, 407 (2011).

12 A. W. W. Ludwig, M. P. A. Fisher, R. Shankar, and G. Grinstein, Phys. Rev. B 50, 7526 (1994).

13 V. P. Gusynin and S. G. Sharapov, Phys. Rev. B 73, 245411 (2006).

14 M. I. Katsnelson, Eur. Phys. J. B 51, 157 (2006).

15 K. Ziegler, Phys. Rev. Lett. 97, 266802 (2006).

16 K. Ziegler, Phys. Rev. B 75, 233407 (2007).

17 V. P. Gusynin, S. G. Sharapov, and J. P. Carbotte, Phys. Rev. Lett. 98, 157402 (2007).

18 L. A. Falkovsky and A. A. Varlamov, Eur. Phys. J. B 56, 281 (2007).

19 T. Stauber, N. M. R. Peres, and A. K. Geim, Phys. Rev. B 78, 085432 (2008).

20 T. G. Pedersen, Phys. Rev. B 79, 113406 (2009).

21 M. Lewkowicz and B. Rosenstein, Phys. Rev. Lett. 102, 106802 (2009).

22 J. J. Palacios, Phys. Rev. B 82, 165439 (2010).

23 L. Moriconi and D. Niemeyer, Phys. Rev. B 84, 193401 (2011).

24 P. V. Buividovich, E. V. Luschevskaya, O. V. Pavlovsky, M. I. Polikarpov, and M. V. Ulybyshev, 
Phys. Rev. B 86, 045107 (2012).

25 Á. Bácsi and A. Virosztek, Phys. Rev. B 87, 125425 (2013).

26 C. A. Dartora and G. G. Cabrera, Phys. Rev. B 87, 165416 (2013).

27 T. Louvet, P. Delplace, A. A. Fedorenko, and D. Carpentier, Phys. Rev. B 92, 155116 (2015).

28 M. Merano, Phys. Rev. A 93, 013832 (2016).

29 Y.-W. Tan, Y. Zhang, K. Bolotin, Y. Zhao, S. Adam, E. H. Hwang, S. Das Sarma, H. L. Stormer, and P. Kim, Phys. Rev. Lett. 99, 246803 (2007).

30 R. R. Nair, P. Blake, A. N. Grigorenko, K. S. Novoselov, T. J. Booth, T. Stauber, N. M. R. Peres, and A. K. Geim, Science 320, 1308 (2008).

31 Z. Li, E. Henriksen, Z. Jiang, Z. Hao, M. Martin, P. Kim, H. Stormer, and D. Basov, Nature Phys. 4, 532 (2008).

32 K. F. Mak, M. Y. Sfeir, Y. Wu, C. H. Lui, J. A. Misewich, and T. F. Heinz, Phys. Rev. Lett. 101, 196405 (2008).

33 J. Horng, C.-F. Chen, B. Geng et al., Phys. Rev. B 83, 165113 (2011).

34 D. Drosdoff and L. M. Woods, Phys. Rev. B 82, 155459 (2010).

35 D. Drosdoff and L. M. Woods, Phys. Rev. A 84, 062501 (2011).

36 Bo E. Sernelius, Europhys. Lett. 95, 57003 (2011).

37 Bo E. Sernelius, Phys. Rev. B 85, 195427 (2012).

38 A. D. Phan, L. M. Woods, D. Drosdoff, I. V. Bondarev, and N. A. Viet, Appl. Phys. Lett. 101, $113118(2012)$.

39 M. Bordag, I. V. Fialkovsky, D. M. Gitman, and D. V. Vassilevich, Phys. Rev. B 80, 245406 (2009).

40 I. V. Fialkovsky, V. N. Marachevsky, and D. V. Vassilevich, Phys. Rev. B 84, 035446 (2011).

41 M. Bordag, G. L. Klimchitskaya, and V. M. Mostepanenko, Phys. Rev. B 86, 165429 (2012).

42 M. Chaichian, G. L. Klimchitskaya, V. M. Mostepanenko, and A. Tureanu, Phys. Rev. A 86, 012515 (2012).

43 G. L. Klimchitskaya and V. M. Mostepanenko, Phys. Rev. B 87, 075439 (2013).

44 G. L. Klimchitskaya, U. Mohideen, and V. M. Mostepanenko, Phys. Rev B 89, 115419 (2014).

45 G. L. Klimchitskaya and V. M. Mostepanenko, Phys. Rev A 89, 052512 (2014).

46 G. L. Klimchitskaya and V. M. Mostepanenko, Phys. Rev. B 91, 045412 (2015).

47 G. L. Klimchitskaya, V. M. Mostepanenko, and Bo E. Sernelius, Phys. Rev. B 89, 125407 (2014). 
48 M. Bordag, G. L. Klimchitskaya, U. Mohideen, and V. M. Mostepanenko, Advances in the Casimir Effect (Oxford University Press, Oxford, 2015).

49 M. Bordag, G. L. Klimchitskaya, V. M. Mostepanenko, and V. M. Petrov, Phys. Rev. D 91, 045037 (2015); Phys. Rev. D 93, 089907(E) (2016).

50 G. L. Klimchitskaya, C. C. Korikov, and V. M. Petrov, Phys. Rev. B 92, 125419 (2015); Phys. Rev. B 93, 159906(E) (2016); G. L. Klimchitskaya and V. M. Mostepanenko, Phys. Rev A 93, 052106 (2016).

51 G. L. Klimchitskaya and V. M. Mostepanenko, Phys. Rev B 91, 174501 (2015).

52 G. L. Klimchitskaya, Int. J. Mod. Phys. A 31, 1641026 (2016).

53 Bo E. Sernelius, J. Phys.: Condens. Matter 27, 214017 (2015).

54 L. A. Falkovsky and S. S. Pershoguba, Phys. Rev. B 76, 153410 (2007).

55 L. D. Landau, E. M. Lifshitz, and L. P. Pitaevskii, Electrodynamics of Continuous Media (Pergamon, Oxford, 1984).

56 T. Stauber, J. Phys.: Condens. Matter 26, 123201 (2014).

57 T. Ando, Y. Zheng, and H. Suzuura, J. Phys. Soc. Jpn. 71, 1318 (2002).

58 N. M. R. Peres and T. Stauber, Int. J. Mod. Phys. B 22, 2529 (2008). 


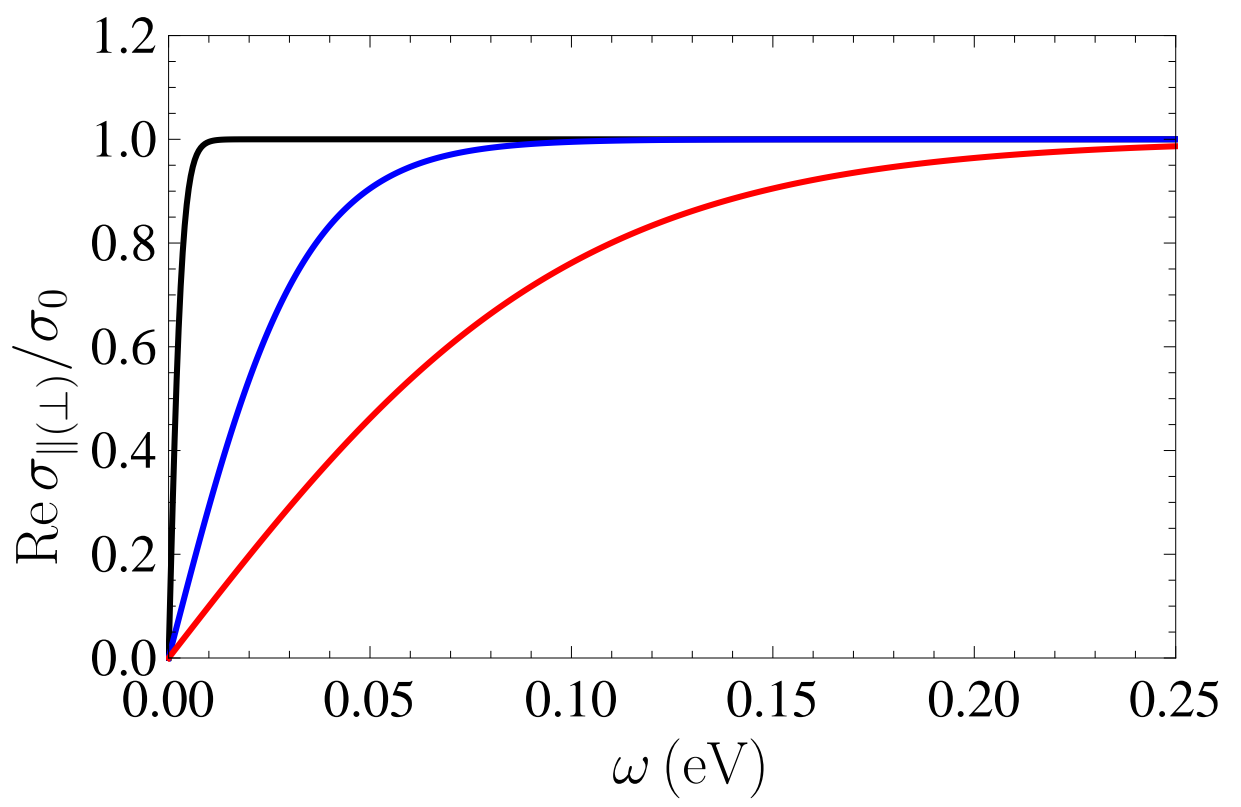

FIG. 1: (Color online) The normalized to $\sigma_{0}$ real part of the conductivity of pure graphene as a function of frequency is shown by the three lines from bottom to top computed at the temperatures $T=300 \mathrm{~K}, 100 \mathrm{~K}$, and $10 \mathrm{~K}$, respectively. 

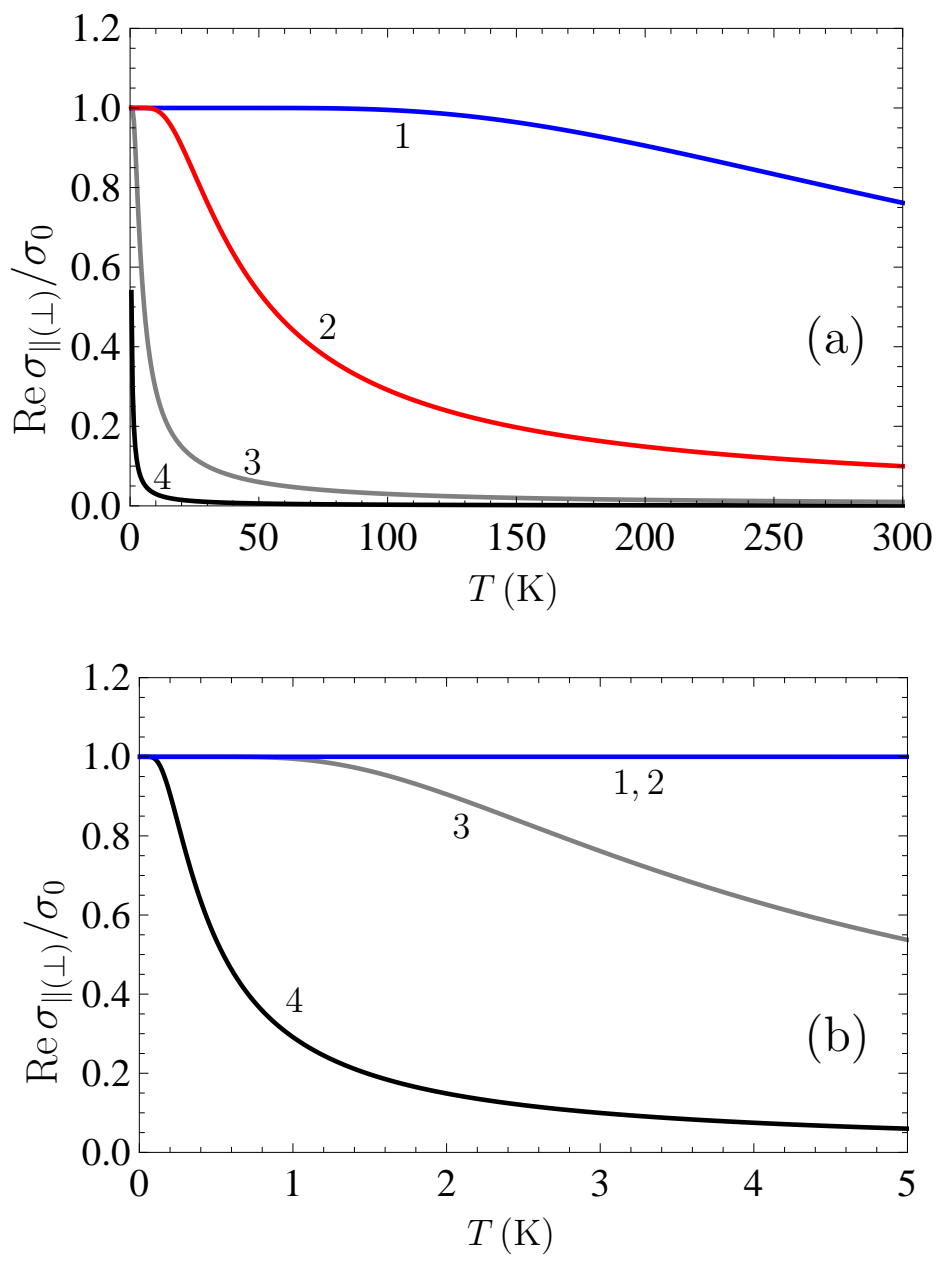

FIG. 2: (Color online) (a) The normalized to $\sigma_{0}$ real part of the conductivity of pure graphene as a function of temperature in the region from $0.5 \mathrm{~K}$ to $300 \mathrm{~K}$ is shown by the lines 1,2 , 3 , and 4 computed at the frequencies $\omega=0.1,0.01,0.001$, and $0.0001 \mathrm{eV}$, respectively. (b) The same results are shown on an enlarged scale in the region from $0 \mathrm{~K}$ to $5 \mathrm{~K}$. 


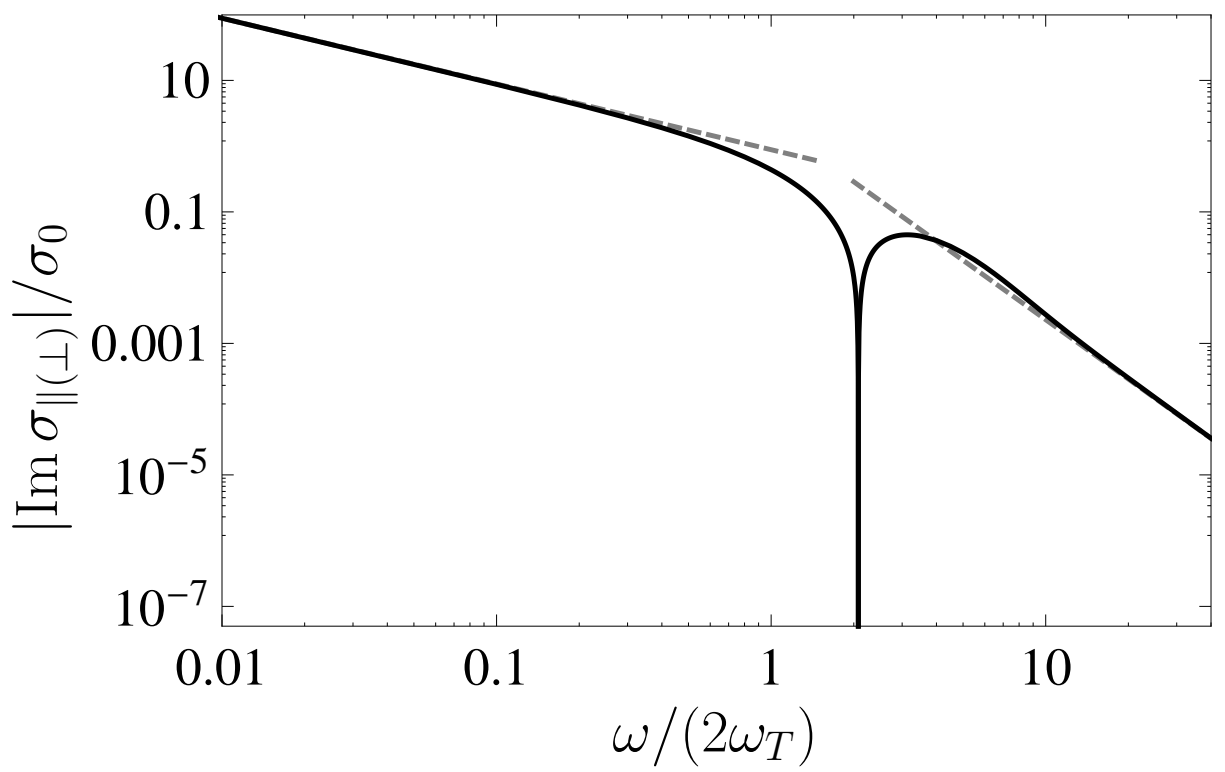

FIG. 3: The normalized to $\sigma_{0}$ magnitude of the imaginary part of the conductivity of pure graphene as a function of the ratio of frequency to twice temperature is shown by the solid line. The left and right dashed lines present the asymptotic results at low and high frequencies, respectively. 


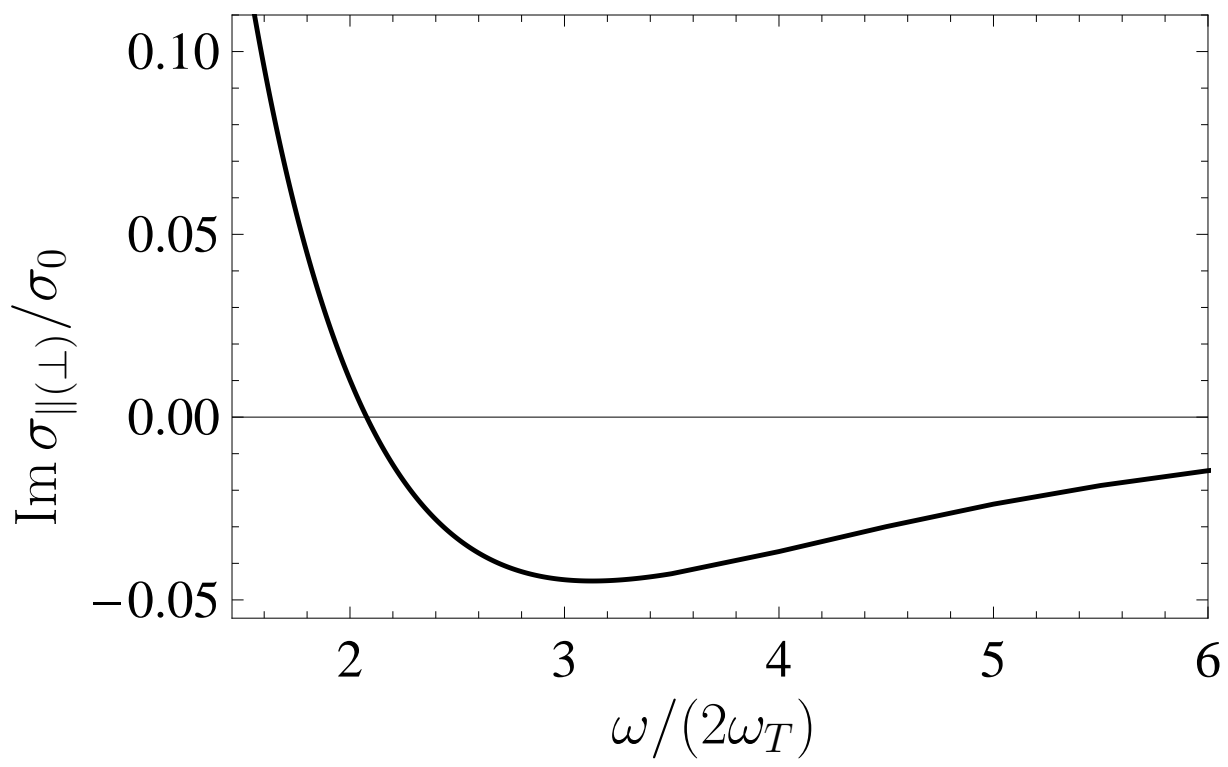

FIG. 4: The normalized to $\sigma_{0}$ imaginary part of the conductivity of pure graphene as a function of the ratio of frequency to twice temperature. 


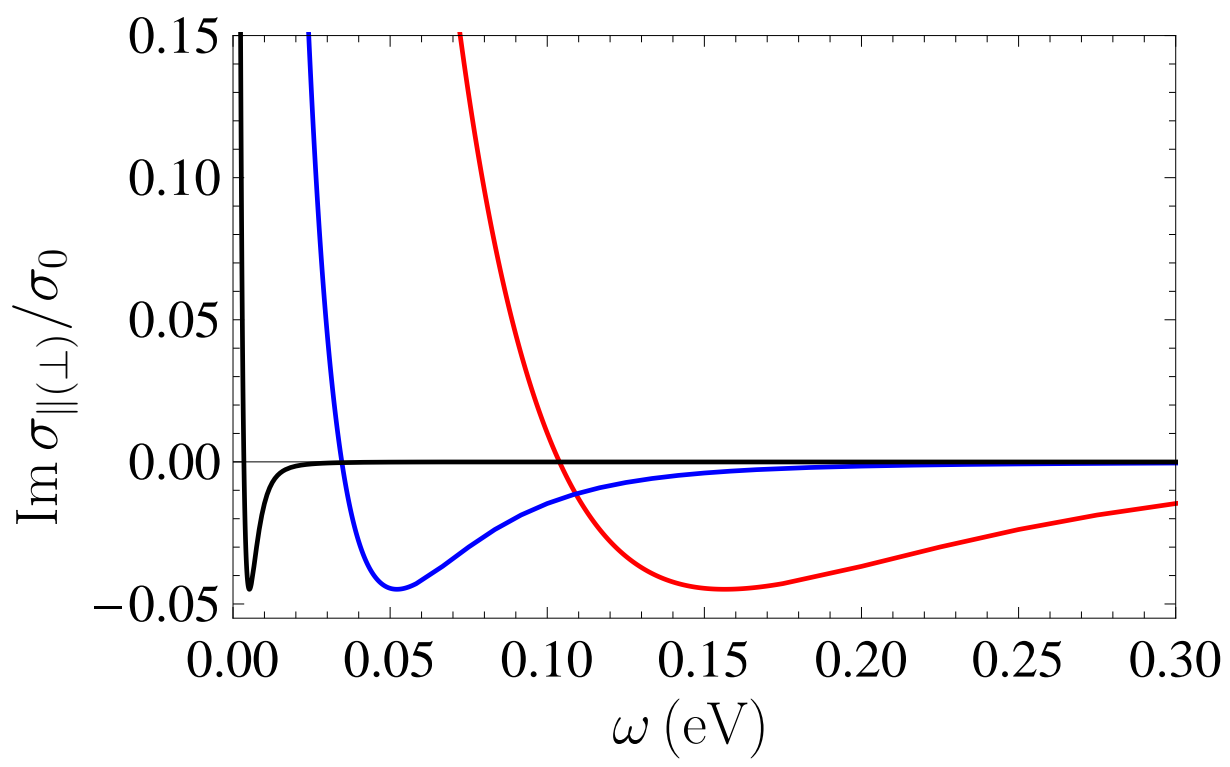

FIG. 5: (Color online) The normalized to $\sigma_{0}$ imaginary part of the conductivity of pure graphene as a function of frequency is shown by the three lines from right to left computed at the temperatures $T=300 \mathrm{~K}, 100 \mathrm{~K}$, and $10 \mathrm{~K}$, respectively. 

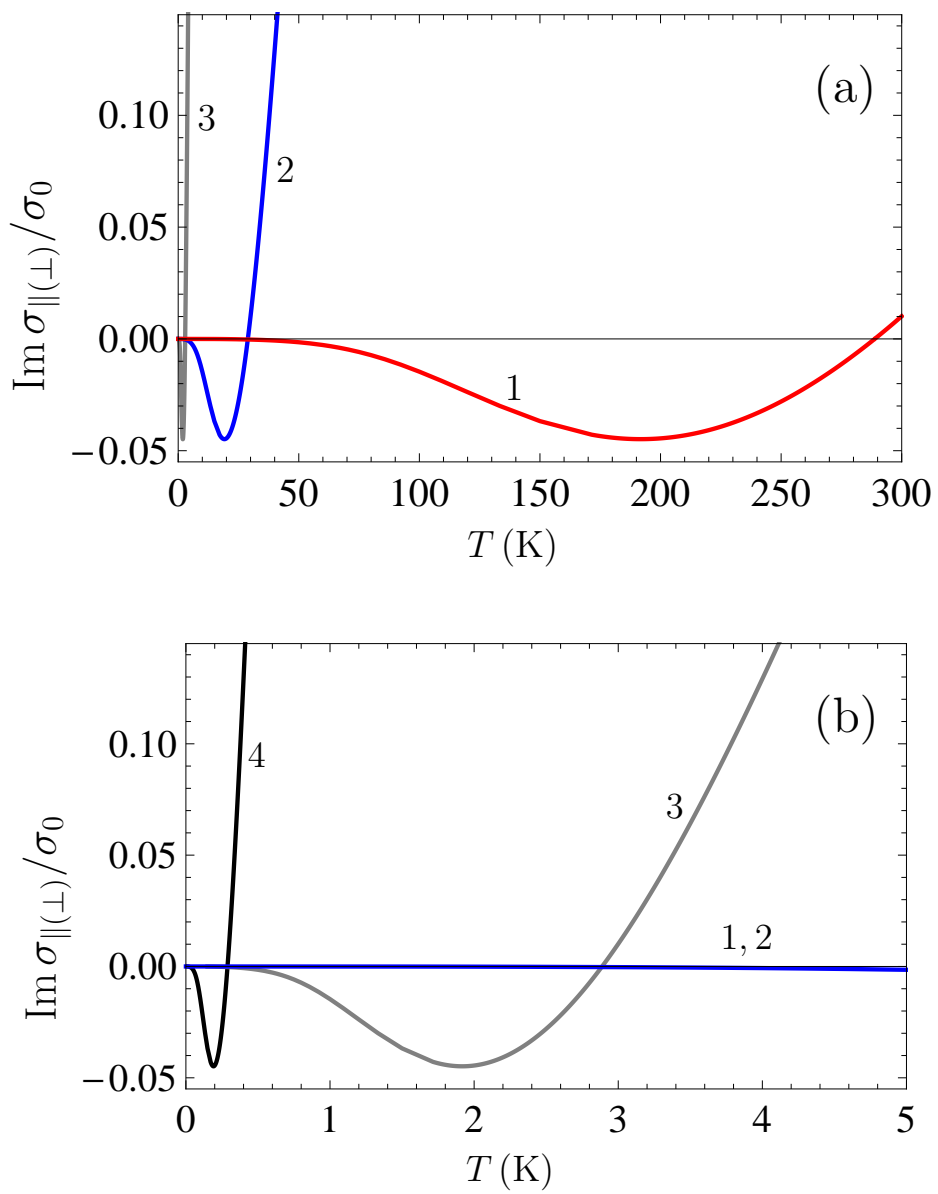

FIG. 6: (Color online) (a) The normalized to $\sigma_{0}$ imaginary part of the conductivity of pure graphene as a function of temperature in the region from $0.5 \mathrm{~K}$ to $300 \mathrm{~K}$ is shown by the lines 1,2 , and 3 computed at the frequencies $\omega=0.1,0.01$, and $0.001 \mathrm{eV}$, respectively. (b) The same results are shown on an enlarged scale in the region from $0 \mathrm{~K}$ to $5 \mathrm{~K}$ and the line 4 is added computed at $\omega=10^{-4} \mathrm{eV}$. 